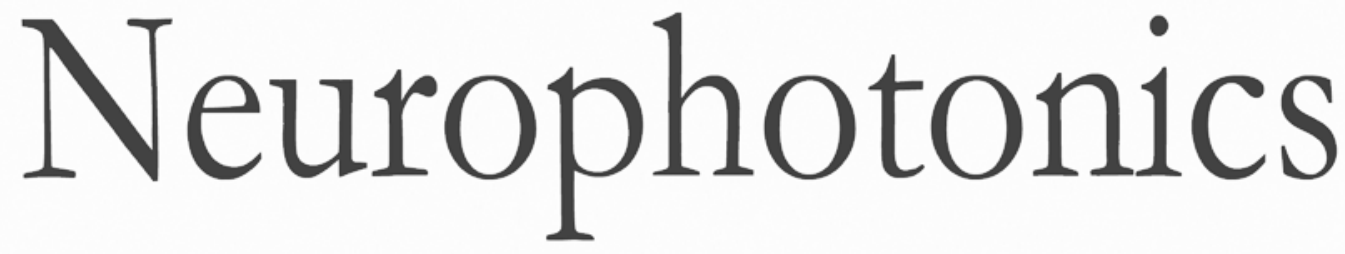

\title{
Rapid, label-free detection of intracranial germinoma using multiphoton microscopy
}

\author{
Na Fang \\ Zanyi Wu \\ Xingfu Wang \\ Ning Cao \\ Yuanxiang Lin \\ Lianhuang Li \\ Yupeng Chen \\ Shanshan Cai \\ Haohua Tu \\ Dezhi Kang \\ Jianxin Chen
}




\title{
Rapid, label-free detection of intracranial germinoma using multiphoton microscopy
}

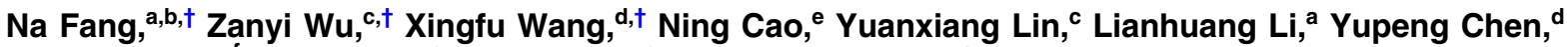 \\ Shanshan Cai, ${ }^{\mathrm{H}}$ Haohua Tu, ${ }^{9}$ Dezhi Kang ${ }^{\mathrm{c}, *}$ and Jianxin Chen ${ }^{\mathrm{a}, \mathrm{g}}$ \\ ${ }^{a}$ Fujian Normal University, Key Laboratory of OptoElectronic Science and Technology for Medicine of Ministry of Education, \\ Fujian Provincial Key Laboratory of Photonics Technology, Fuzhou, China \\ ${ }^{\text {b} F u j i a n ~ M e d i c a l ~ U n i v e r s i t y, ~ D e p a r t m e n t ~ o f ~ O p h t h a l m o l o g y ~ a n d ~ O p t o m e t r y, ~ F u z h o u, ~ C h i n a ~}$ \\ ${ }^{c}$ The First Affiliated Hospital of Fujian Medical University, Department of Neurosurgery, Fuzhou, China \\ ${ }^{\mathrm{d} T}$ The First Affiliated Hospital of Fujian Medical University, Department of Pathology Fuzhou, China \\ eZhangzhou Affiliated Hospital of Fujian Medical University, Department of Plastic Surgery, Zhangzhou, China \\ 'The Second Affiliated Hospital of Fujian Medical University, Department of Pathology Quanzhou, China \\ 'University of Illinois at Urbana-Champaign, Beckman Institute for Advanced Science and Technology, Urbana, Illinois, United States
}

\begin{abstract}
Accurate histopathological diagnosis is essential for facilitating the optimal surgical management of intracranial germinoma. Current intraoperative histological methods are time- and labor-intensive and often produce artifacts. Multiphoton microscopy (MPM) is a label-free imaging technique that can produce intraoperative histological images of fresh, unprocessed surgical specimens. We employ an MPM based on second-harmonic generation and two-photon excited fluorescence microscopy to image fresh, unfixed, and unstained human germinoma specimens. We show that label-free MPM is not only capable of identifying various cells in human germinoma tissue but also capable of revealing the characteristics of germinoma such as granuloma, stromal fibrosis, calcification, as well as the abnormal and uneven structures of blood vessels. In conjunction with custom-developed image-processing algorithms, MPM can further quantify and characterize the extent of stromal fibrosis and calcification. Our results provide insight into how MPM can deliver rapid diagnostic histological data that could inform the surgical management of intracranial germinoma. $\odot$ The Authors. Published by SPIE under a Creative Commons Attribution 4.0 Unported License. Distribution or reproduction of this work in whole or in part requires full attribution of the original publication, including its DOI. [DOI: 10.1117/1.NPh.6.3.035014]
\end{abstract}

Keywords: intracranial germinoma; multiphoton microscopy; two-photon excited fluorescence; second-harmonic generation.

Paper 19039RR received Apr. 12, 2019; accepted for publication Aug. 28, 2019; published online Sep. 27, 2019.

\section{Introduction}

The accurate intraoperative histopathological diagnosis of intracranial germinoma is critical for determining the most appropriate surgical treatment, prognosis assessment, and predicting or evaluating a given patient's response to a particular therapeutic approach. ${ }^{1}$ The histopathology of frozen sections using hematoxylin and eosin (H\&E) staining has been the gold standard for the definitive diagnosis of intracranial germinoma during brain tumor surgery. For decades, this technique has been considered to be tried and true. However, despite encouraging results, this method has several limitations, such as its inapplicability in situ, the potential for sampling errors, crush artifacts, bleeding, associated costs, labor-intensity, and the time-consuming nature of the procedure $(\sim 30 \mathrm{~min}))^{2,3}$ Consequently, it would be highly desirable to develop a technology for performing real-time histological diagnoses during brain surgeries.

In recent years, confocal laser endomicroscopy with subcellular resolution has emerged as a noninvasive on-site brain tissue imaging tool, enabling the in-vivo diagnosis of brain tissue., ${ }^{4,5}$ This technique can produce histological images of tissues while avoiding the risks and costs associated with conventional biopsies. However, exogenous fluorescent agents must be applied to provide sufficient contrast for highlighting the

*Address all correspondence to Dezhi Kang, E-mail: kdz99988@vip.sina.com; Jianxin Chen, E-mail: chenjianxin@fjnu.edu.cn

tThese authors contributed equally to this work. structure and physiology of nuclei, cytoplasms, and vasculature. Multiphoton microscopy (MPM) based on two-photon excited fluorescence (TPEF) and second-harmonic generation (SHG) is a more robust imaging tool that has attracted much attention since its inception. ${ }^{6-11}$ This imaging modality is capable of producing images with a level of detail similar to that of standard histological procedures by detecting intrinsic fluorescence chromophores while demonstrating superior tissue penetration depth and reduced degrees of photobleaching and photodamage. Owing to these significant advantages over confocal laser microscopy, MPM is widely applied in the investigation of healthy versus diseased brain tissues. ${ }^{12-17}$

In this exploratory analysis, we first assessed MPM's capability of characterizing pathological features associated with intracranial germinoma and comparing the yielded images to gold standard histopathology. Second, using custom-developed image-processing algorithms, we then assessed MPM's capability of quantifying the extent of stromal fibrosis and calcification in intracranial germinoma, which are the two most significant factors that affect surgeries. To the best of our knowledge, no other study has been conducted around the use of MPM for examining human intracranial germinoma.

\section{Materials and Methods}

\subsection{Samples Preparation}

Every patient signed an informed consent in advance in this research. The research had been approved by Fujian Medical 
Table 1 Clinical report of the patients involved in this study.

\begin{tabular}{lccccccr} 
& & & \multicolumn{2}{c}{ Number of tissue studied } & & Primary or \\
Patient no. & Sex & Age & Normal & Tumor & & Location of tumor & recurrence status \\
\hline 1 & Male & 7 & 1 & 1 & Hypothalamus & First resection \\
2 & Female & 8 & 1 & 1 & Hypothalamus & First resection \\
3 & Male & 10 & 1 & 1 & Hypothalamus & First resection \\
4 & Male & 21 & 1 & 1 & Hypothalamus & First resection \\
5 & Female & 5 & 1 & 1 & Pineal body & First resection \\
6 & Male & 8 & 0 & 1 & Pineal body & First resection \\
7 & Female & 27 & 0 & & Recurrence \\
\hline
\end{tabular}

University Clinical Research Screening Committee for Studies Involving Human Subjects. From 2015 to 2018, the fresh human normal brain and intracranial germinoma samples were obtained during the conventional diagnosis process in the Neurosurgery Department at The First Affiliated Hospital of Fujian Medical University. Table 1 shows the clinic-pathological features of the subjects. The samples removed by the surgeons were delivered to the pathology lab and then processed into five serial slices at about $20 \mu \mathrm{m}$ of thickness by cryostat microtome. In this context, the middle of the slice was processed with H\&E staining for the histological imaging, and the remaining slices were sandwiched between a coverslip and a microscope slide for the MPM imaging. The pathological diagnosis results for germinoma specimens and normal human brain tissue specimens were separately confirmed by two professional neuropathologists.

\subsection{Multiphoton Microscopic Imaging System}

MPM system used in this study had been elaborated previously. ${ }^{18,19}$ Briefly, the system was composed of a Zeiss LSM 880 system (Jena, Germany) and a Ti:sapphire femtosecond laser $(140 \mathrm{fs}, 80 \mathrm{MHz})$ tuned from 690 to $1064 \mathrm{~nm}$ (Chameleon Ultra, Coherent, Inc., Santa Clara, California). A $63 \times$ Plan-Apochromat oil immersion objective [numerical aperture (NA) $=1.4$, Zeiss, Jena, Germany] and 10× PlanApochromat objective (NA $=0.45$, Zeiss, Jena, Germany) were used for focusing the excitation beam on the samples and collecting the TPEF and backscattered SHG signals. The $10 \times$ PlanApochromat objective was capable of acquiring the whole sample images and the $63 \times$ Plan-Apochromat objective was capable of acquiring detailed images of region of interest. Simultaneously, two different channels were selected to examine the TPEF/SHG signals from intracranial germinoma specimens. TPEF signals were detected by one channel, covering from 430 to $690 \mathrm{~nm}$; SHG signals were detected through another channel, covering from 395 to $415 \mathrm{~nm}$. TPEF signals were detected through 32-channel GaAsP PMT (Zeiss, Jena, Germany) array detector, which can collect emission signals from 410 to $690 \mathrm{~nm}$ at intervals of $8.9 \mathrm{~nm}$, whereas SHG signals were detected through a flanking PMT detector (Zeiss, Jena, Germany). To highlight the contrasts of TPEF/SHG images, TPEF images were marked as red color and SHG images as green color. The images were obtained at the depth of 12-bit pixels.
In this study, the excitation wavelength of $810 \mathrm{~nm}$ was chosen. For 810-nm excitation, the nicotinamide adenine dinucleotide (NAD) and flavin adenine dinucleotide (FAD) in the cells, the elastin in the blood vessel wall, as well as collagen in stroma and blood vessel wall, were all excited and the signal-to-noise ratio of these signals was optimal.

\subsection{Quantification Analysis of Collagen Density in Intracranial Germinoma}

In our experiment, the customized program was applied to automatically assess the extent of stromal fibrosis in intracranial germinoma. A program was developed to analyze and segment MPM images through MATLAB Image Processing Toolbox (The MathWorks, Inc.). To automatically measure collagen content (the ratio of the pixels of collagen to all pixels in every MPM image), MPM images were processed as follows. First, the SHG image was converted to grayscale image. Next, a Gaussian filter $($ size $=5$, sigma $=1$ ) was used to reduce noise and enhance the collagen details of image. Third, the Otsu threshold was applied to segment the image, and the closed operation of morphological filtering method was used to further extract the edges of collagen. Finally, the segmentation template was obtained, and the collagen density was calculated according to the occupied area of the collagen, as shown in Fig. 1(a). (a)

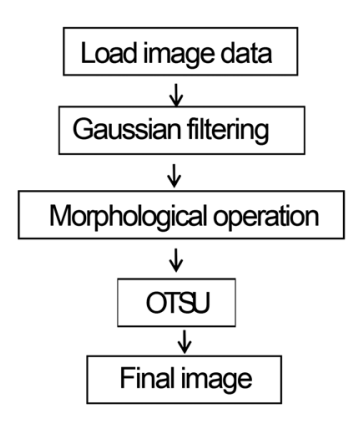

(b)

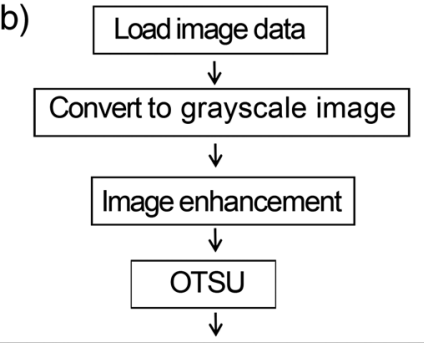

Remove small connected regions

$\downarrow$

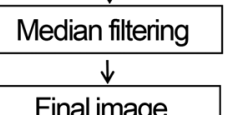

Fig. 1 (a) Flowchart of the proposed collagen density segmentation and (b) flowchart of the proposed calcification density segmentation. 


\subsection{Quantification Analysis of Calcification Density in Intracranial Germinoma}

To further measure the calcification content (the ratio of the pixels of calcification to all pixels in every MPM image), another program was developed and MPM images were processed as follows. First, the original MPM image was converted to a grayscale image and then image gray value was mapped to the entire range of gray level by gray stretch transformation, in order to increase the contrast of the image. Extending the range of gray level made the calcification features more obvious. Second, the Otsu threshold was applied to segment the image. Third, the segmentation binary image was processed by removing small connected regions and median filtering to further reduce noise. Finally, the segmentation template was obtained, and the calcification density was calculated according to the occupied area of the calcification, as shown in Fig. 1(b).

\section{Results}

\subsection{Multiphoton Microscopy Imaging in the Normal Brain Tissue}

Figure 2 displayed the typical MPM images and corresponding $\mathrm{H} \& \mathrm{E}$ images in the normal brain tissues (in hypothalamus). Figures 2(e)-2(1) show higher magnifications of the selected regions [marked as cyan and white square boxes in Fig. 2(c)]. In the large-area images [Figs. 2(a)-2d)], the extracellular matrix, the cells, and the blood vessels that were the major brain tissue components emitted strong multiphoton signals, which might be explicitly distinguished in MPM images. More details of the cells and blood vessels were revealed in the magnified images [Figs. 2(e)-2(1)]. The NAD (phosphate) hydrogen [NAD(P)H] and FAD in the cytoplasm emitted a strong TPEF signal and is presented as a red color while cell nuclei were lacking fluorescent signals and appeared as dark spots, which created a shadow contrast image with a clearly distinguishable cell (cyan arrowheads). A blood vessel (purple arrowheads) could be recognized, owing to its tubeshaped structures determined by the elastin and collagen. Except for the blood vessel wall, no other SHG signals were detected, indicating that the collagen contents were limited in the stroma of normal brain tissues. The results were consistent with previous reports. ${ }^{20}$ These characteristics were also present in the corresponding H\&E-stained image. However, the collagen in H\&E-stained images could not be displayed as clearly as that in MPM images.

\subsection{Multiphoton Microscopy Imaging of Intracranial Germinoma}

Compared with normal brain tissues, germinoma tissues had the unique morphological features, for example, a biphasic
Large-area images

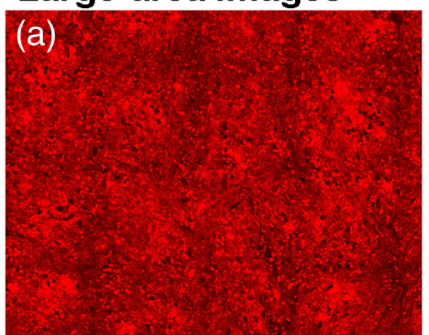

Magnified images
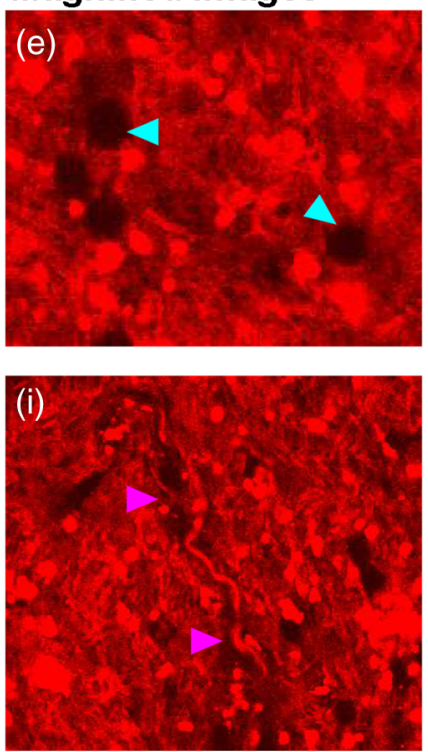
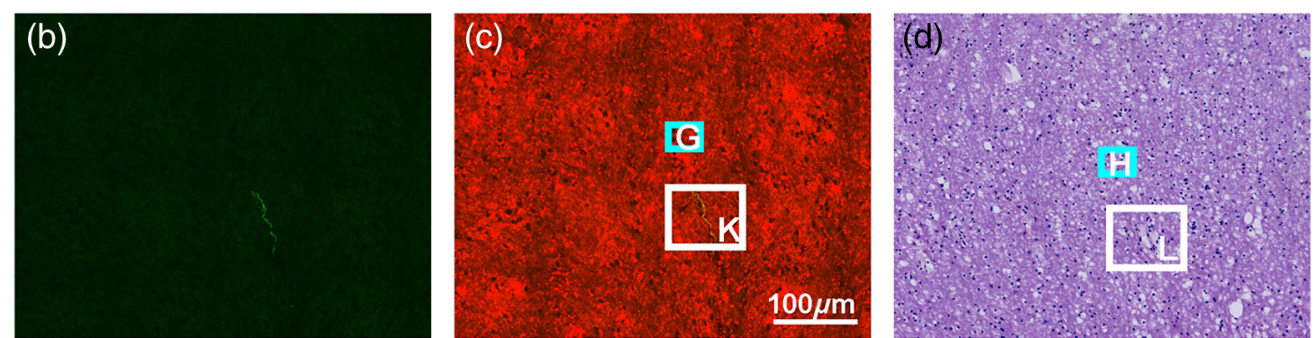

Cells
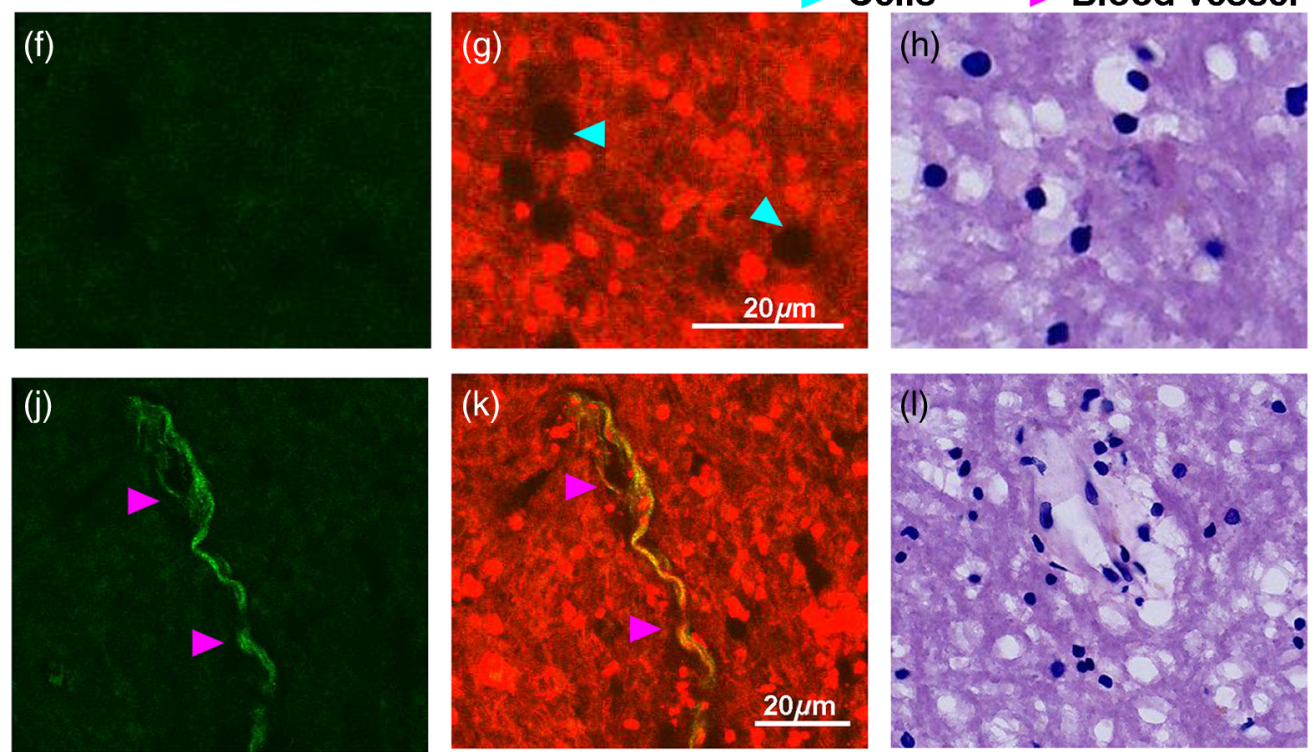

Fig. 2 Representative MPM images and the corresponding H\&E-stained images of normal brain (white matter). (a)-(d) TPEF, SHG, TPEF/SHG overlaid, and corresponding H\&E-stained image of normal brain tissue. (e)-(h) The magnified TPEF, SHG, TPEF/SHG overlaid, and corresponding H\&E image of the selected area (cyan square box) in Fig. 3(c). (i)-(I) The magnified TPEF, SHG, TPEF/SHG overlaid, and corresponding H\&E image of the selected area (white square box) in Fig. 3(c). Cyan arrowheads: cells in normal brain tissue. Purple arrowheads: blood vessel in normal brain tissue. 


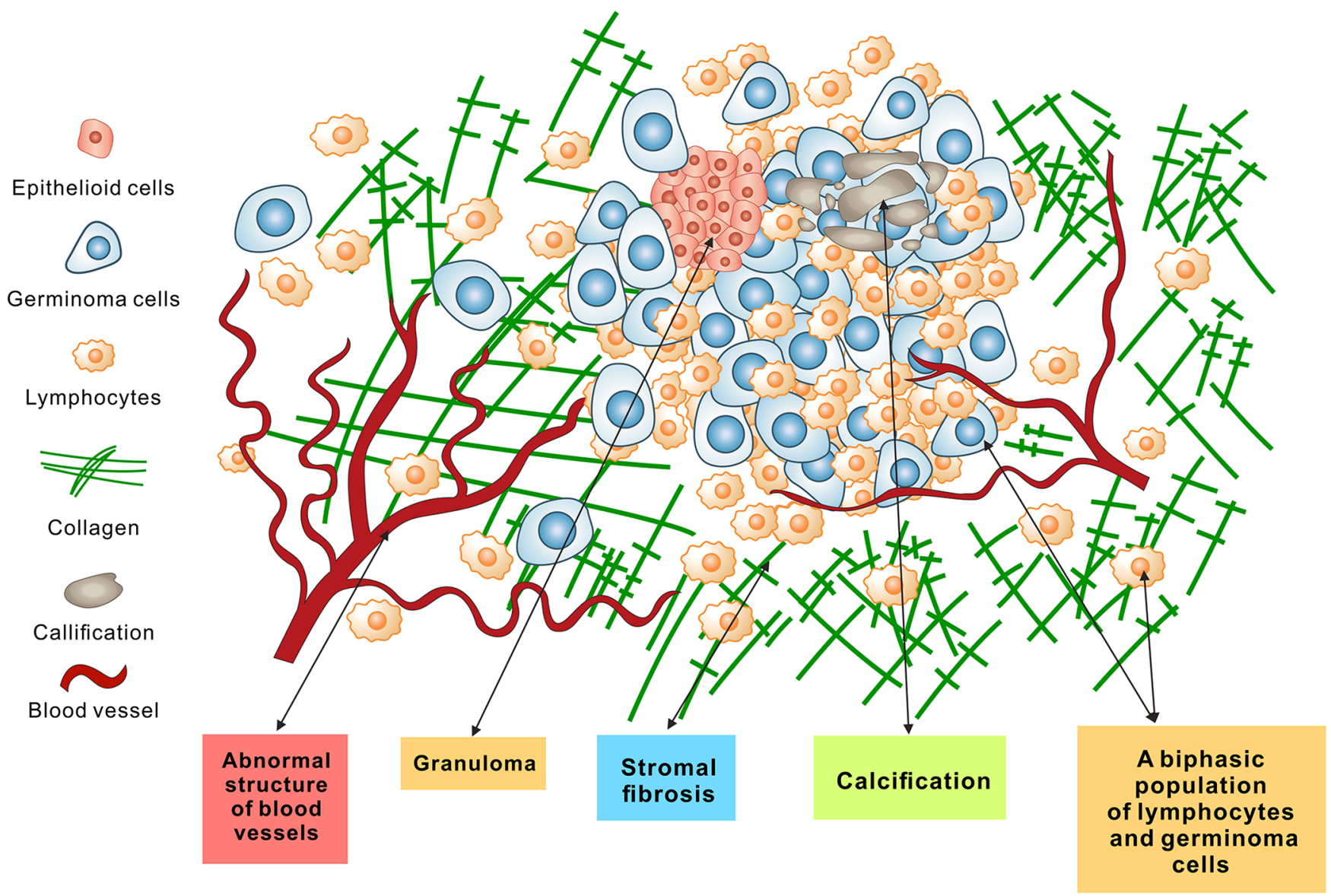

Fig. 3 Schematic illustration of morphological characteristics of germinoma.

population of lymphocytes and germinoma cells, granuloma, stromal fibrosis, calcification, as well as abnormal and uneven vascular structures, as shown in Fig. 3.

These features were quite important for the diagnosis of intracranial germinoma, which could be exactly identified in the MPM images, as shown in Figs. 4-6, respectively.

In Fig. 4, MPM images show that the cell number dramatically increased in the intracranial germinoma tissues; in addition, the cells of various morphology and sizes were well characterized by TPEF signals. Figures 4(e)-4(t) [marked as cyan, yellow, and white square boxes in Fig. 4(c)] show magnified images for the chosen regions in Fig. 4(c). In the magnified images, more details were revealed. The morphological difference of germinoma cells and lymphocytes were clearly displayed as well [Figs. 4(e) and 4(i)]. Although both germinoma cells (green arrowheads) and lymphocytes (cyan arrowheads) had distinct cell boundaries, the arrangement and size of the nuclei were quite different. When compared with germinoma cells (20 to $30 \mu \mathrm{m}$ ), the lymphocytes had more compact arrangements but smaller nucleus $(\sim 10 \mu \mathrm{m})$. MPM identification of the biphasic population of lymphocytes and larger germinoma cells made it possible for cytological diagnosis of intracranial germinoma. ${ }^{21}$

Except for a biphasic population of lymphocytes and germinoma cells, granuloma was another significant diagnostic feature of intracranial germinoma. Granuloma was composed of clumps of epithelioid cells. Figures $4(m)-4(p)$ show the microstructure of granuloma (yellow arrowheads) in intracranial germinoma. A cluster of cells (blue arrowheads) with uniform nuclear size and shape were displayed in Figs. 4(m) and 4(q). Those cell boundaries in the TPEF image were not distinctly revealed, unlike those of tumor cells and lymphocyte cells. Meanwhile, epithelioid cells have a unique arrangement. Usually they gather together to form a cluster structure, which can help distinguish them from other cells. Those morphological features of the nuclei were consistent with those in the corresponding H\&E-stained images. Compared with H\&E-staining technique, MPM had more advantages in the mapping of cytoplasm.

Stromal fibrosis, another morphological feature of intracranial germinoma, could be clearly delineated in SHG images, as shown in Fig. 4 (the second row). Unlike in the normal brain tissue, collagen content was sharply increased in intracranial germinoma. Some collagen fibers (purple arrowheads) were in beam arrangement and some in mesh arrangement. Here, the collagen fibers were found circled by lymphocyte cells. However, there were no or few collagen fibers surrounding the tumor cells and epithelioid cells of granuloma. Nevertheless, the patterns could not be explicitly depicted by the corresponding H\&E-stained images.

Calcification, another common morphological feature of intracranial germinoma, is illustrated in Fig. 5. Figures 5(a)-5(d) display the large-area MPM image and corresponding H\&Estained images of calcification region, and Figs. 5(e)-5(l) [marked as cyan and white square boxes in Figs. 5(c)] show the magnified image for the chosen region in Fig. 5(c). In the large-area images, the calcification region could be clearly identified because stronger TPEF signals could be emitted than those 
Large-area images

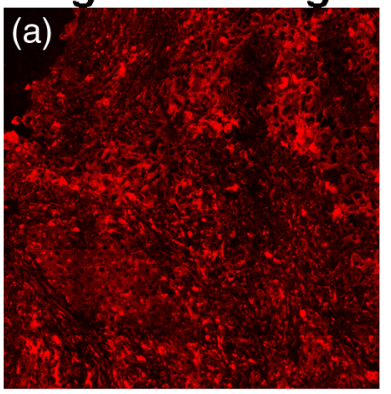

Magnified images
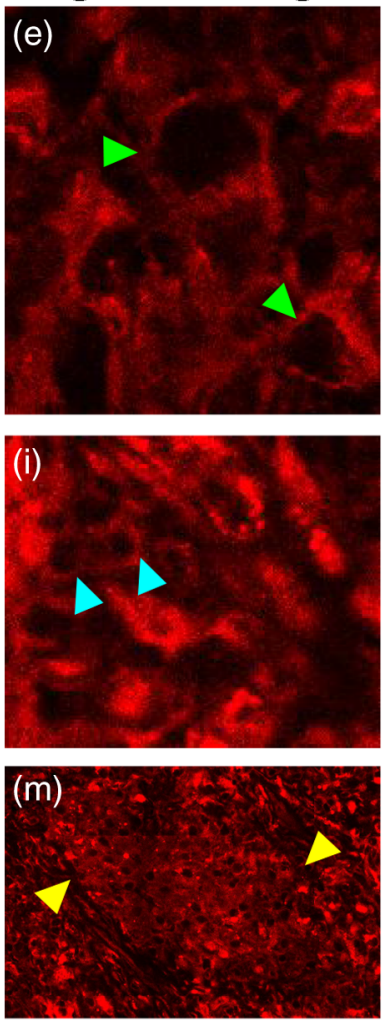

Magnified images

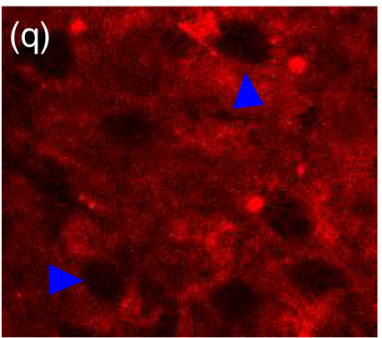

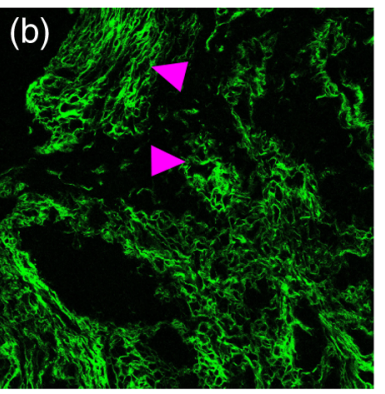
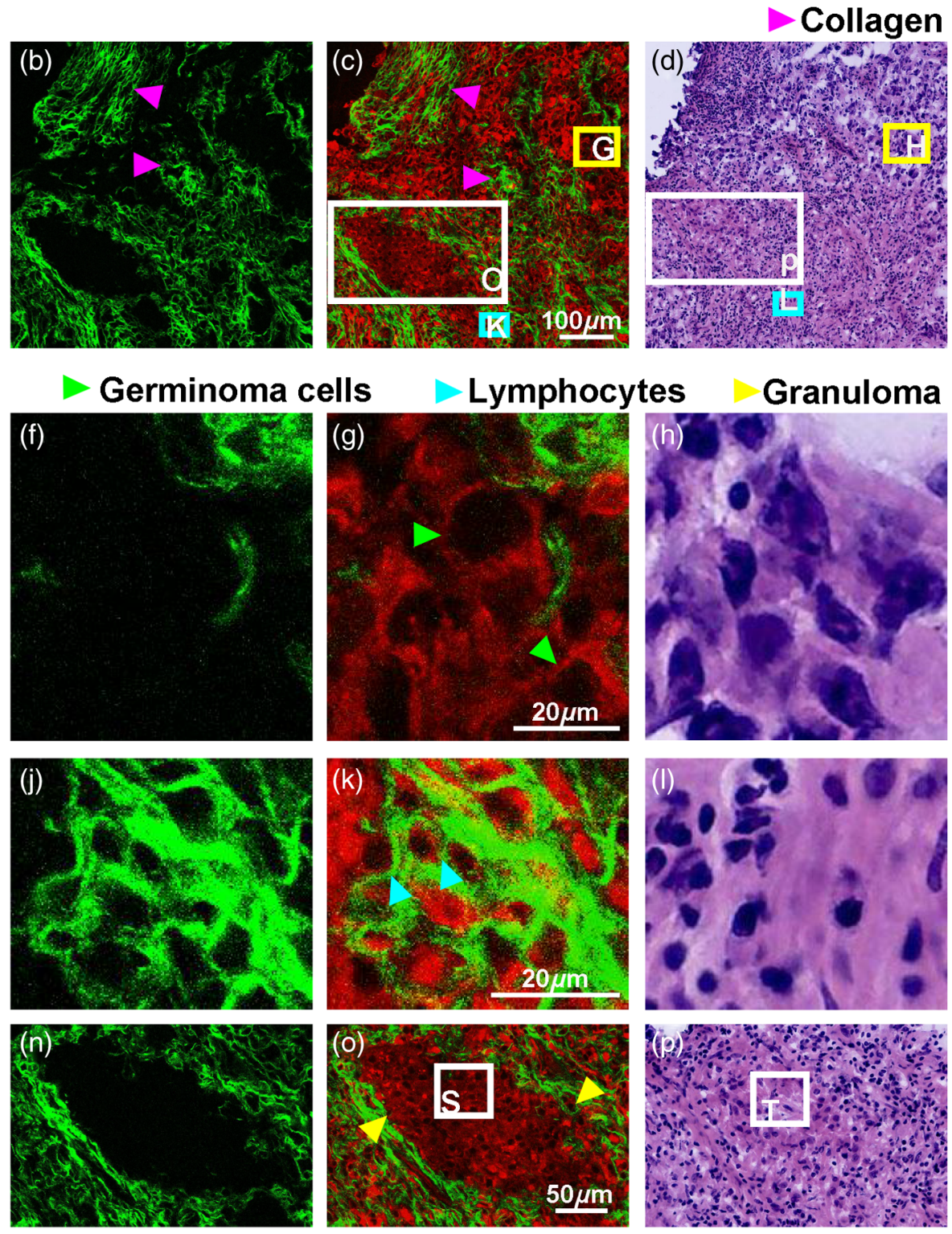

\section{Epithelioid cells}
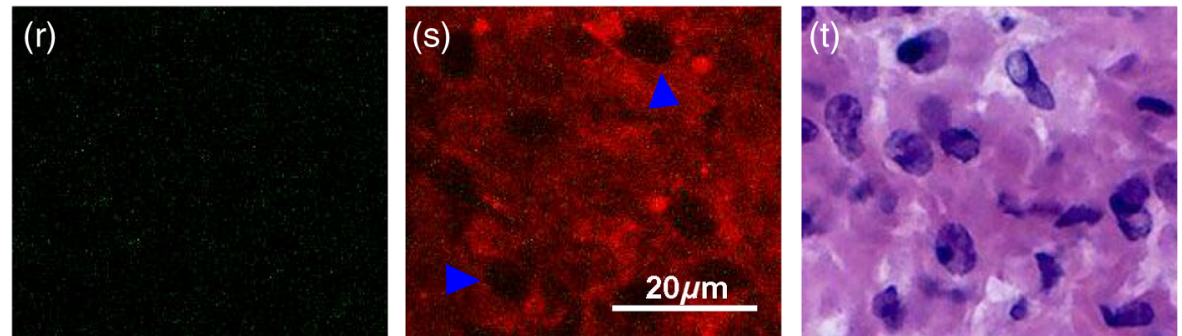

Fig. 4 Representative MPM images and the corresponding H\&E-stained images of intracranial germinoma with a biphasic population of lymphocytes and germinoma cells, stromal fibrosis and granuloma. (a)-(d) TPEF, SHG, TPEF/SHG overlaid, and corresponding H\&E-stained image of intracranial germinoma. (e)-(h) The magnified TPEF, SHG, TPEF/SHG overlaid, and corresponding H\&E image of the selected area (yellow square box) in (c). (i)-(l) The magnified TPEF, SHG, TPEF/SHG overlaid, and corresponding $\mathrm{H} \& E$ image of the selected area (cyan square box) in Fig. $3(\mathrm{c}) .(\mathrm{m})-(\mathrm{p})$ The magnified TPEF, SHG, TPEF/SHG overlaid and corresponding H\&E image of the selected area (white square box) in (c). (q)-(t) The magnified TPEF, SHG, TPEF/SHG overlaid, and corresponding H\&E image of the selected area (white square box) in (o). Purple arrowheads: collagen; green arrowheads: germinoma cells; cyan arrowheads: lymphocytes; yellow arrowheads: granuloma; and blue arrowheads: epithelioid cells. 


\section{Large-area images}
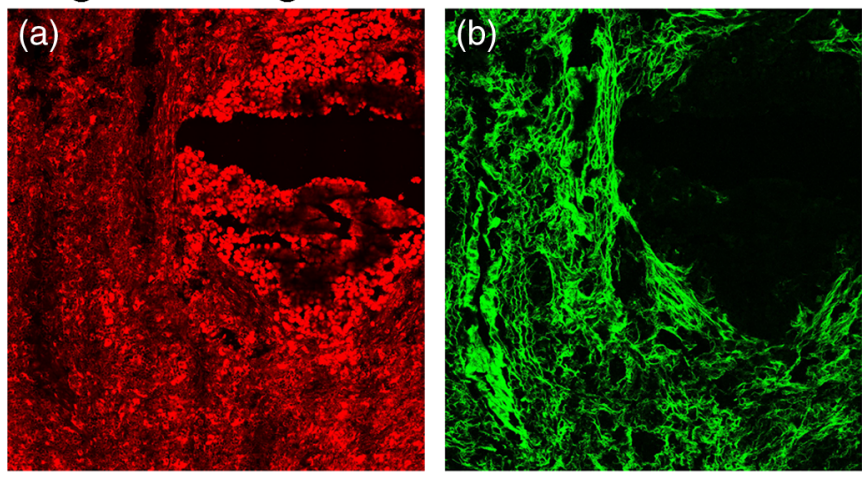

\section{Magnified images}
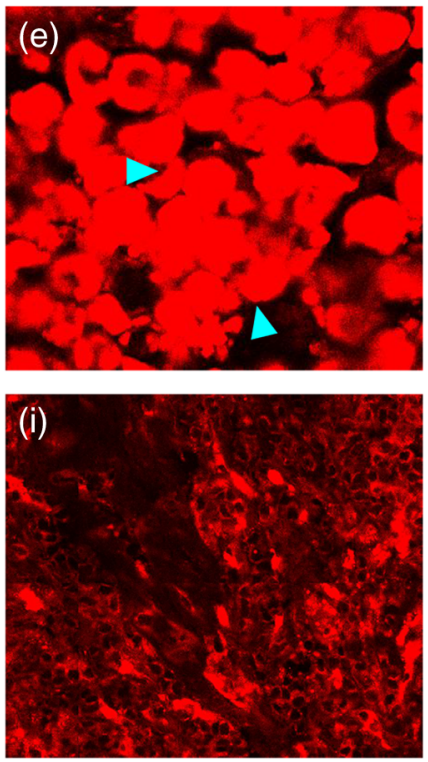
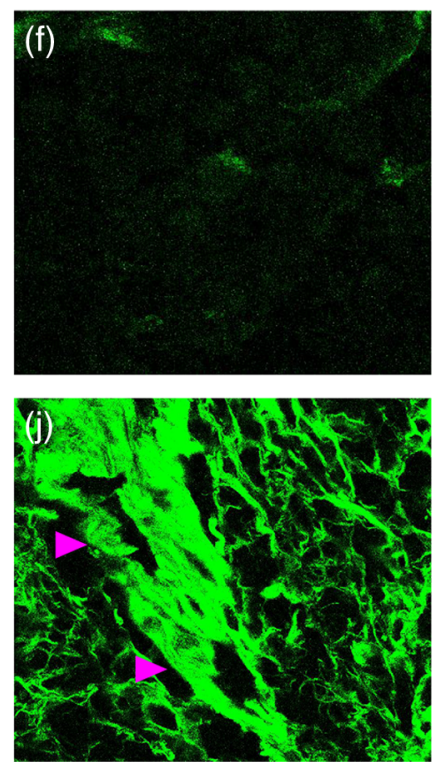
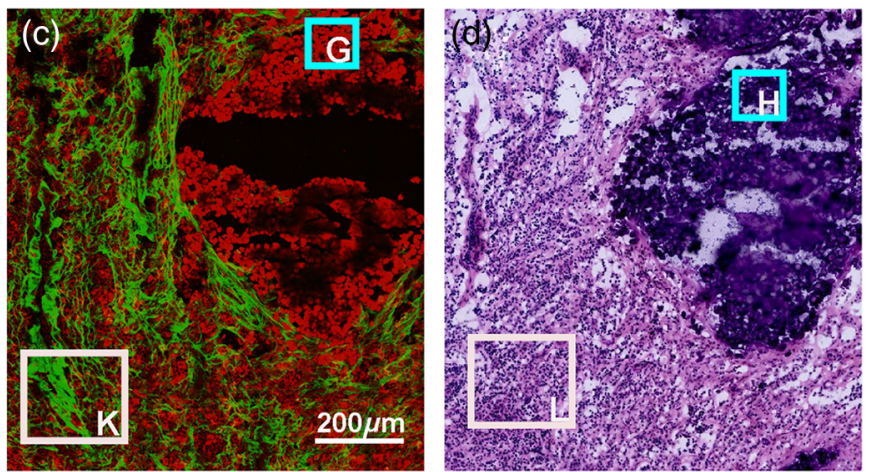

Calcification Collagen bundle
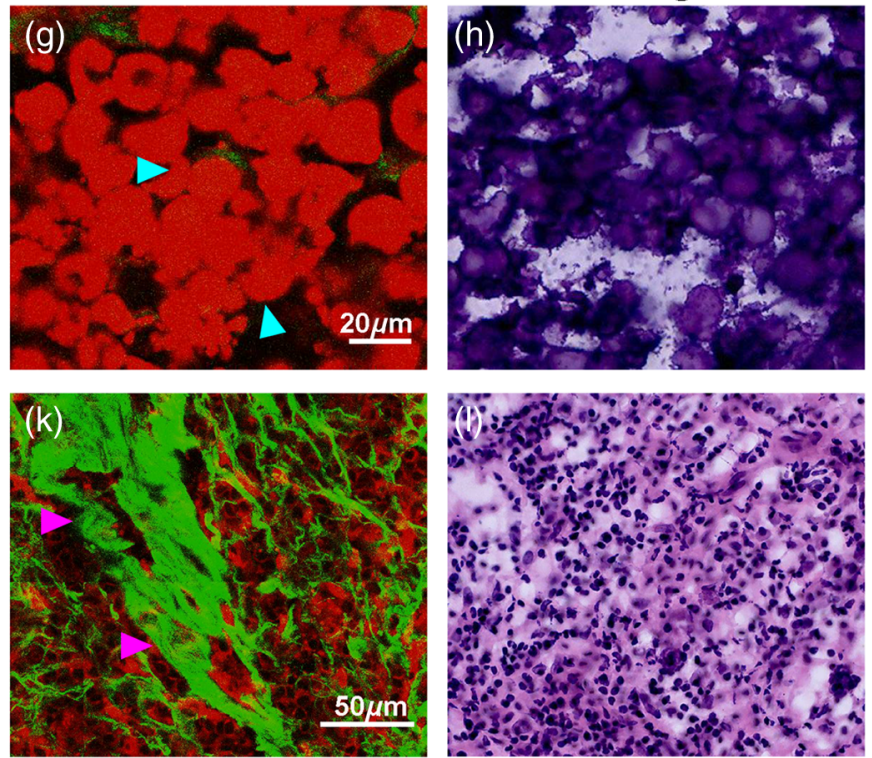

Fig. 5 Representative MPM images and the corresponding H\&E-stained images of intracranial germinoma with calcification. (a)-(d) TPEF, SHG, TPEF/SHG overlaid, and corresponding H\&E-stained image of intracranial germinoma with calcification. (e)-(h) The magnified TPEF, SHG, TPEF/SHG overlaid, and corresponding H\&E image of the selected area (cyan square box) in (c). (i)-(I) The magnified TPEF, SHG, TPEF/SHG overlaid, and corresponding H\&E image of the selected area (white square box) in (c). Cyan arrowheads: calcification. Purple arrowheads: collagen bundle.

in the surrounding cells. More morphological details of the calcification region (cyan arrowheads) were provided in the magnified images. Notably, although many collagen fibrils were found deposited in the stroma and some were even condensed into collagen bundles (purple arrowheads), generating strong SHG signals, no collagen fibers were detected in the calcification region. Those morphological calcification details were in perfect agreement with the corresponding H\&E-stained images, but the collagen deposition in the stroma was not clearly depicted in H\&E-stained image.

The abnormal and uneven structure of blood vessels in intracranial germinoma was another main feature. ${ }^{21}$ The tube-shaped and capillary-shaped blood vessels were generally regular, thin, and small in the normal brain tissues. However, blood vessels of different morphology and sizes in intracranial germinoma tissues were seriously dilated and deformed. Figures 6(a)-6(d) show large-area MPM images and corresponding H\&E-stained images of intracranial germinoma with dilated blood vessel, and Figs. 6(e)-6(p) [marked as cyan, yellow, and white square boxes in Fig. 6(c)] show the magnified image of the chosen region in Fig. 6(c). In the large-area images, a large blood vessel (purple arrowheads) could be readily identified. In the blood vessel wall, the elastin, collagen, and proliferation cells were clearly displayed in the magnified image. In some regions, the cavity of the blood vessel was filled with those proliferation (cyan arrowheads) cells, resulting in the vascular leakage and edema and irregular blood flow and avoiding the systemic delivery of chemotherapeutics to treat cancer. ${ }^{22,23}$ Interestingly, more collagen fibers appeared on the right side of the blood vessel than that on the left side. In the magnified image, we found that more lymphocytes (yellow arrowheads) existed in the abundant collagen fiber region, whereas more tumor cells (green arrowheads) were found in the few collagen fiber regions. The results demonstrated that there might be a close relationship between collagen deposition and lymphocyte aggregation in intracranial germinoma. Those morphological details corrected well with the corresponding H\&E-stained image, but collagen deposition of surround region could not clearly be depicted in H\&E-stained images.

Above results demonstrated that MPM made it possible to show and visualize the morphological characteristics of intracranial germinoma just like those of histological examination. 

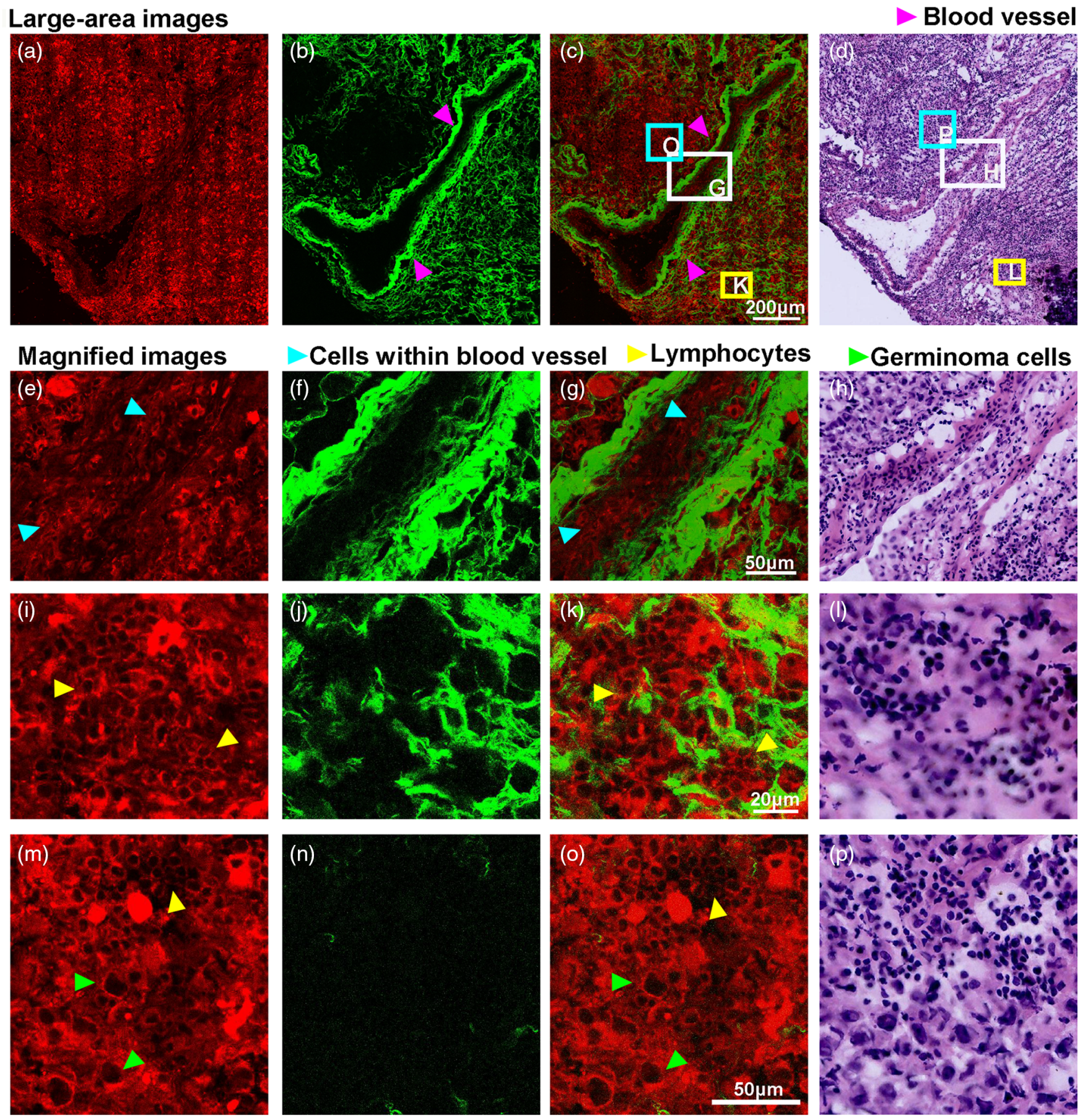

Fig. 6 Representative MPM images and the corresponding H\&E-stained images of intracranial germinoma with large dilated blood vessel. (a)-(d) TPEF, SHG, TPEF/SHG overlaid, and corresponding H\&Estained image of intracranial germinoma with large dilated blood vessel. (e)-(h) The magnified TPEF, SHG, TPEF/SHG overlaid, and corresponding H\&E image of the selected area (white square box) in (c). (i)-(I) The magnified TPEF, SHG, TPEF/SHG overlaid, and corresponding H\&E image of the selected area (yellow square box) in (c). (m)-(p) The magnified TPEF, SHG, TPEF/SHG overlaid, and corresponding H\&E image of the selected area (cyan square box) in (c). Purple arrowheads: blood vessel; cyan arrowheads: cells within vascular wall; yellow arrowheads: lymphocytes; and green arrowheads: germinoma cells.

To a limited extent, better effects were presented in MPM, when compared with those of histological examination. Furthermore, MPM technique could capture the image features faster than the pathologists. Figure 7 shows MPM images and corresponding H\&E-stained images for the whole sample; it just took $8 \mathrm{~min}$ for the MPM to capture the images, which was much faster than the fast intraoperative frozen section technique $(\sim 30 \mathrm{~min})$ that was the golden standard for diagnosis of intracranial germinoma. As a whole, MPM quite fitted for the morphological diagnosis of intracranial germinoma. 

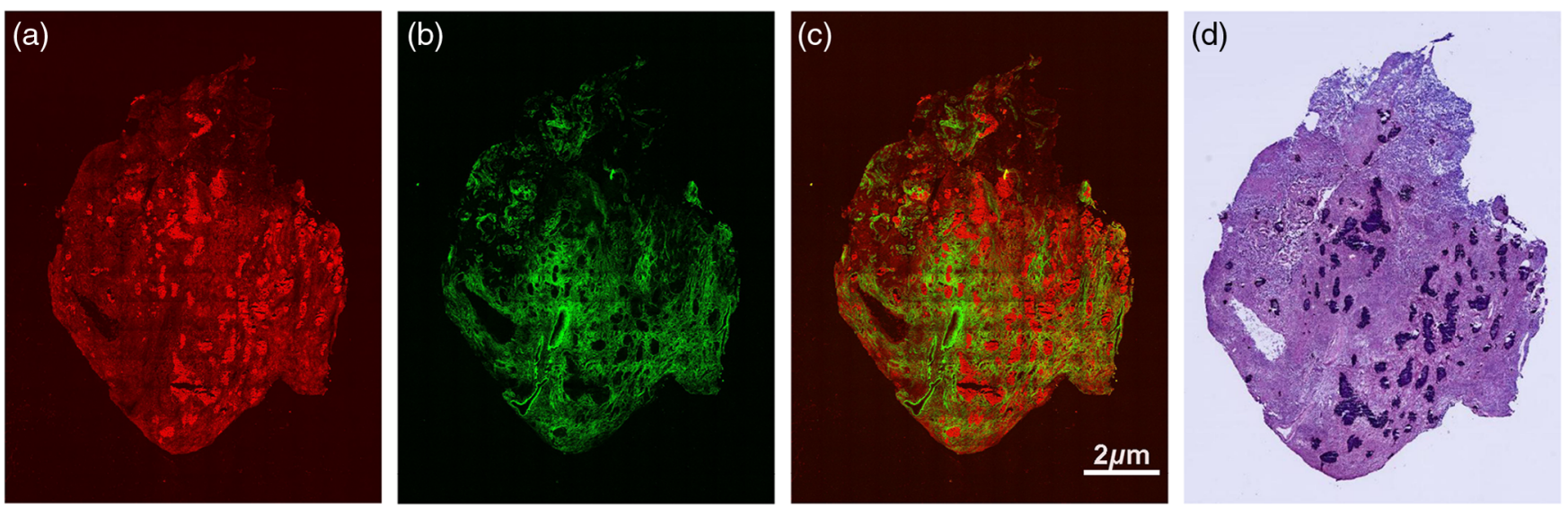

Fig. 7 Representative MPM images and the corresponding H\&E-stained images of a whole intracranial germinoma sample. (a) TPEF image of a whole sample. (b) SHG image of a whole sample. (c) TPEF/ SHG overlaid image of a whole sample. (d) Corresponding H\&E-stained image of a whole sample.

\subsection{Automatic Calculation of Collagen Content in Intracranial Germinoma}

Quantitative analysis was conducted to further depict the collagen characteristics in intracranial germinoma. A program (Refer Sec. 2.3) was established to automatically calculate collagen content in intracranial germinoma. Figure 8 shows the representative segmentation results of the collagen in the whole sample. In this program, the collagen could be positioned and then collagen density would be automatically calculated. Figures 8(a)-8(d) show the detailed segmentation results of the collagen. The images for collagen enhancement [Fig. 8(b)], collagen position segmentation [Fig. 8(c)], and final segmentation [Fig. 8(d)] were positively associated with SHG images
[Fig. 8(a)] at the subcellular level. Figures 8(e)-8(h) show the segmentation of another image with different level of fibrosis, further suggesting that the collagen algorithm can work. The quantitative analysis results showed that the collagen content of Figs. 8(a) and 8(e) were 0.30484 and 0.12961 , respectively, while their corresponding evaluation times in the program were only 2.3 and $2.1 \mathrm{~s}$, respectively.

\subsection{Automatic Calculation of Calcification Density in Intracranial Germinoma}

To quantitatively analyze the extent of calcification in intracranial germinoma, another program (Refer Sec. 2.4) was made to automatically count calcification content in intracranial
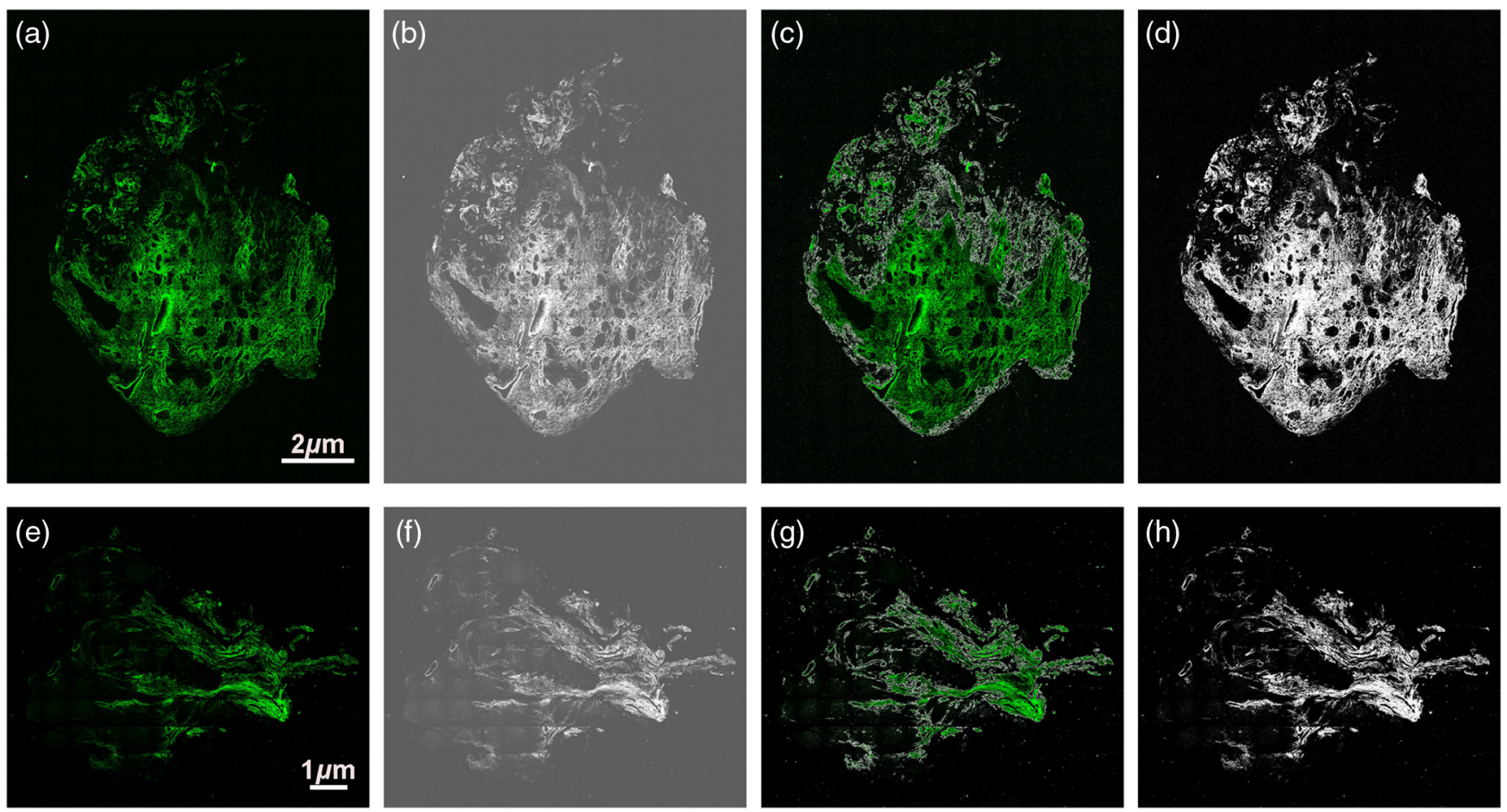

Fig. 8 Quantitative analysis of collagen in intracranial germinoma. (a), (e) Original SHG image.

(b), (f) The result after image enhancement. (c), (g) The segmentation result of collagen position.

(d), (h) The final segmentation result of collagen content. 

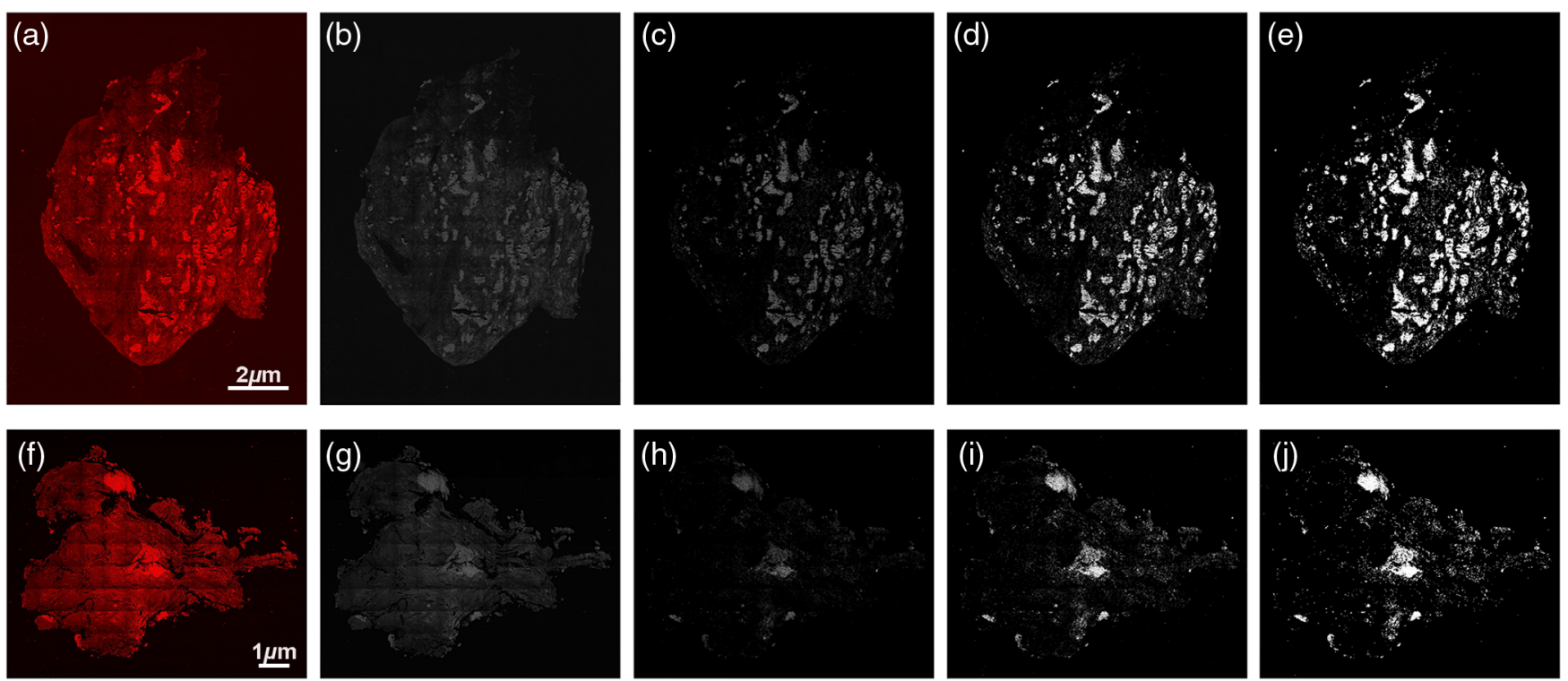

Fig. 9 Quantitative analysis of calcification in intracranial germinoma. (a), (f) an original TPEF image. (b), (g) The result after converting to grayscale image. (c), (h) The result after image enhancement operation. (d), (i) The result after Otsu segmentation. (e), (j) The final segmentation result of calcification content.

germinoma. Figure 9 shows the segmentation consequences of calcification region. In Fig. 9(b), it is seen that the original TPEF [Fig. 9(a)] is converted into grayscale image. After image enhancement segmentation, the rough boundary of calcification region was sketched out [Fig. 9(c)]. After Otsu segmentation, the grayscale image was converted to the binary image, and the rough boundary of calcification region could be delineated [Fig. 9(d)]. After removing small connected regions operation and median filtering operation were further carried out, a more precise boundary of calcification region was obtained, and the final segmentation consequences of calcification region are shown in Fig. 9(e). Figures 9(f)-9(j) show the segmentation of another image with a different level of calcification, further suggesting that the calcification algorithm can work. The quantitative analysis results showed that the calcification content of Figs. 8(a) and 8(e) were 0.191324 and 0.089737 , respectively, while the corresponding evaluation times in the program were only 3.7 and $3.3 \mathrm{~s}$, respectively. The comparison results about the collagen density and between normal brain and intracranial germinoma using collagen analysis method and calcification analysis method are shown in Table 2. The quantitative analysis results showed that the collagen and calcification content in intracranial germinoma were all much higher than those in normal brain tissue. Those results showed that the combination of MPM and image-processing method allowed the rapid,

Table 2 Quantitative data in normal brain and intracranial germinoma.

\begin{tabular}{lcccccc} 
& \multicolumn{2}{c}{ Collagen content } & & \multicolumn{2}{c}{$\begin{array}{c}\text { Calcification } \\
\text { content }\end{array}$} \\
\cline { 2 - 3 } \cline { 6 - 7 } Sample & Mean & SD & & Mean & SD \\
\hline Normal brain & 0.005 & 0.003 & & 0 & 0 \\
Intracranial germinoma & 0.237 & 0.073 & & 0.125 & 0.109 \\
\hline
\end{tabular}

automatic, and quantitative analysis about the extent of stromal fibrosis and calcification in intracranial germinoma.

\section{Discussion}

In this study, MPM imaging and H\&E staining are compared and evaluated in a comprehensive way. Our results indicate that MPM technology can efficiently identify germinoma from the normal tissues. MPM has the potential to obtain the high-resolution and high-contrast images in germinoma and normal brain tissues. The research findings indicate that MPM imaging can be used to examine many diagnostic hallmarks, which are important to the identification of intracranial germinoma. In addition, MPM imaging can provide similar details data such as from H\&E-stained frozen sections at large scale. Owing to the sharp contrast between nonfluorescence nuclei, fluorescence cytoplasm and cell arrangement, different cells are clearly visualized and easily identified by MPM techniques. Therefore, a biphasic population of lymphocytes and germinoma cells as well as granuloma can be readily identified by MPM. Moreover, the calcification region can be easily detected by MPM techniques, because stronger TPEF signals can be emitted by comparison with its surrounding cells.

In addition, we are able to visualize the collagen that cannot be well characterized by standard H\&E-stained sections. Based on this, the abnormal structure of blood vessels can be obviously detected by MPM. Notably, MPM imaging of fresh germinoma tissues allows us to calculate the amount of collagen deposited in the stroma that cannot be detected by standard H\&E-staining technique.

In this study, with the image analysis, MPM techniques also allow us to automatically measure the collagen density and calcification density. Previous reports have demonstrated that the extent of stroma fibrosis and calcification are the main influence factors during the total resection of brain tumor tissues. ${ }^{24,25}$ Therefore, imaging and the quantification of collagen density and calcification density before and during brain surgery is of great importance. In this context, MPM combined with image 
processing can be a favorable method for the neuropathologist to quickly and effectively assess the extent of stromal fibrosis and calcification and decide the most appropriate surgical methods and therapeutic schemes.

It is worth mentioning that the SHG signals were collected backward and the visualized collagen is mesh structure in our work. Previous reports have shown that SHG is clearly sensitive to the organized collagen such as those that are parallel or are formed into bundles, ${ }^{26,27}$ whereas, it is not clear how sensitive it is to mesh collagen where the orientations are more randomized. Collagen fluorescence is strong for cross-linked collagen but weak when collagen around tumors has its cross-links broken down. ${ }^{28}$ That is, we only visualize and automatically calculate backward-sensitive collagen, and some cross-linked brokendown collagen may not be present in our MPM images. Interestingly, in our pilot study, we found that there is less collagen around the tumor cells, suggesting that the production of collagenases may be the reason for this, which has been suggested to have a role in the breakdown of collagen cross-links in the stromal matrix and facilitating tumor cell migration. In our next work, what collagen MPM technique is sensitive or insensitive to and by how much will be further studied.

In short, the rapid and label-free MPM imaging has the potential to provide pathology-like data with obvious contrasts and high sensitivity in human germinoma tissues. The technology allows the rapid intraoperative diagnosis of germinoma just like the detailed histology images while without the tissue removal and staining. In addition, MPM provides insights into the diagnostic features that cannot be displayed by $\mathrm{H} \& \mathrm{E}$ staining. Our results provide insight into how MPM can deliver rapid diagnostic data that could inform the surgical management of germinoma.

\section{Disclosures}

The authors declare that there are no conflicts of interest related to this article.

\section{Acknowledgments}

The project was supported by the Joint Funds of Fujian Provincial Health and Education Research (Grant No. WKJ2016-2-28); the National Natural Science Foundation of China (Grant No. 81671730); the Program for Changjiang Scholars and Innovative Research Team in University (Grant No. IRT_15R10); and Special Funds of the Central Government Guiding Local Science and Technology Development (Grant No. 2017L3009).

\section{References}

1. L. Eisenhardt and H. Cushing, "Diagnosis of intracranial tumors by supravital technique," Am. J. Pathol. 6(5), 541-552 (1930).

2. A. Perry and D. J. Brat, Practical Surgical Neuropathology: A Diagnostic Approach, Churchill Livingstone, London (2010).

3. Y. Uematsu et al., "The usefulness and problem of intraoperative rapid diagnosis in surgical neuropathology," Brain Tumor Pathol. 24(2), 47-52 (2007).

4. N. Sanai et al., "Intraoperative confocal microscopy for brain tumors: a feasibility analysis in humans," Oper. Neurosurg. (Hagerstown) 68(suppl_2), 282-290 (2011).

5. J. Eschbacher et al., "In vivo intraoperative confocal microscopy for real-time histopathological imaging of brain tumors," J. Neurosurg. 116(4), 854-860 (2012).

6. J. Chen et al., "Multiphoton microscopic imaging of histological sections without hematoxylin and eosin staining differentiates carcinoma in situ lesion from normal oesophagus," Appl. Phys. Lett. 103(18), 183701 (2013).

7. W. R. Zipfel et al., "Live tissue intrinsic emission microscopy using multiphoton-excited native fluorescence and second harmonic generation," Proc. Natl. Acad. Sci. U. S. A. 100(12), 7075-7080 (2003).

8. X. Zhu et al., "Monitoring wound healing of elastic cartilage using multiphoton microscopy," Osteoarthritis Cartilage 21(11), 1799-1806 (2013).

9. S. W. Perry, R. M. Burke, and E. B. Brown, "Two-photon and second harmonic microscopy in clinical and translational cancer research," Ann. Biomed. Eng. 40(2), 277-291 (2012).

10. J. W. Birk et al., "Second harmonic generation imaging distinguishes both high-grade dysplasia and cancer from normal colonic mucosa," Dig. Dis. Sci. 59(7), 1529-1534 (2014).

11. D. A. Dombeck et al., "Uniform polarity microtubule assemblies imaged in native brain tissue by second-harmonic generation microscopy," Proc. Natl. Acad. Sci. U. S. A. 100(12), 7081-7086 (2003).

12. J. Leppert et al., "Multiphoton excitation of autofluorescence for microscopy of glioma tissue," Neurosurgery 58(4), 759-767 (2006).

13. S. R. Kantelhardt et al., "Imaging of brain and brain tumor specimens by time-resolved multiphoton excitation microscopy ex vivo," NeuroOncology 9(2), 103-112 (2007).

14. S. R. Kantelhardt et al., "Multi-photon excitation fluorescence microscopy of brain-tumour tissue and analysis of cell density," Acta Neurochir. 151(3), 253-262 (2009).

15. S. R. Kantelhardt et al., "In vivo multiphoton tomography and fluorescence lifetime imaging of human brain tumor tissue," J. Neuro-Oncol. 127(3), 473-482 (2016).

16. L. Jiang et al., "Label-free imaging of brain and brain tumor specimens with combined two-photon excited fluorescence and second harmonic generation microscopy," Laser Phys. Lett. 14(10), 105401 (2017).

17. M. Zanello et al., "Multimodal optical analysis of meningioma and comparison with histopathology," J. Biophotonics 10(2), 253-263 (2017).

18. S. Wang et al., "Optical visualization of cerebral cortex by label-free multiphoton microscopy," IEEE J. Sel. Top. Quantum Electron. 25(1), 1-8 (2019).

19. S. Wang et al., "Rapid, label-free identification of cerebellar structures using multiphoton microscopy," J. Biophotonics 10(12), 1617-1626 (2017).

20. L. S. Payne and P. H. Huang, "The pathobiology of collagens in glioma," Mol. Cancer Res. 11(10), 1129-1140 (2013).

21. D. N. Louis et al., "The 2007 WHO classification of tumours of the central nervous system," Acta Neuropathol. 114(2), 97-109 (2007).

22. R. K. Jain, "Molecular regulation of vessel maturation," Nat. Med. 9(6), 685-693 (2003).

23. G. Helmlinger et al., "Solid stress inhibits the growth of multicellular tumor spheroids," Nat. Biotechnol. 15(8), 778-783 (1997).

24. H. Naganuma, E. Satoh, and H. Nukui, "Technical considerations of transsphenoidal removal of fibrous pituitary adenomas and evaluation of collagen content and subtype in the adenomas," Neurol. Med. Chir. 42(5), 202-213 (2002).

25. H. L. Weiner et al., "Craniopharyngiomas: a clinicopathological analysis of factors predictive of recurrence and functional outcome," Neurosurgery 35(6), 1001-1011 (1994).

26. G. Cox et al., "3-dimensional imaging of collagen using second harmonic generation," J. Struct. Biol. 141(1), 53-62 (2003).

27. G. Cox et al., "Characterization of the second harmonic signal from collagen," Proc. SPIE 4963, 32-40 (2003).

28. D. I. Zeugolis et al., "An in situ and in vitro investigation for the transglutaminase potential in tissue engineering," J. Biomed. Mater. Res. A 92(4), 1310-1320 (2010).

Na Fang received her master's degree in optics from Fujian Normal University, Fuzhou, China, in 2008. Currently, she is working toward her PhD in optics at Fujian Normal University, Fuzhou, China, where she is working with the Institute of Laser and Optoelectronics Technology. Her main research interests include the development and applications of nonlinear optical microscopy in brain and biomedical research.

Zanyi Wu received his master's degree in surgery from Fujian Medical University, Fuzhou, China, in 2007. Currently, he is an 
associate chief physician at the Neurosurgery Department, The First Affiliated Hospital of Fujian Medical University. His main research interests include brain tumor surgery and electrophysiology.

Xingfu Wang received his master's degree in pathology from Fujian Medical University, Fuzhou, China, in 2008. Currently, he is an associate professor of pathology and neuropathologist at The First Affiliated Hospital of Fujian Medical University. His research interests include neuropathology and surgical pathology.

Ning Cao received her master's degree in plastic surgery from Fujian Medical University, Fuzhou, China, in 2007. Currently, she is working in the Zhangzhou Affiliated Hospital of Fujian Medical University. Her research interests include laser cosmetology and diagnosis and treatment of surface malignant tumors.

Yuanxiang Lin received his MD degree in clinical medicine from the Department of Neurosurgery, Southern Medical University, Guangzhou, China, in 2006. He is the chief director of the Neurosurgery Department at The First Affiliated Hospital of Fujian Medical University, Fuzhou, China. Meanwhile, he is a professor and a master-student adviser at Fujian Medical University. His expertise is brain tumor surgery and treatment of functional encephalopathy.

Lianhuang Li received his doctoral degree in physics from Fujian Normal University, Fuzhou, China, in 2016. Currently, he is working at Fujian Provincial Key Laboratory for Photonics Technology, Key Laboratory of Optoelectronic Science and Technology for Medicine of Ministry of Education, Fujian Normal University. His main research interests include the development and applications of nonlinear optical microscopy.
Yupeng Chen received his bachelor's degree in pathology from Fujian Medical University, Fuzhou, China, in 2010. Currently, he is working at The First Affiliated Hospital of Fujian Medical University. His research interests include neuropathology and surgical pathology.

Shanshan Cai received her master's degree in pathology from Fujian Medical University, Fuzhou, China, in 2013. She is now working at the Second Affiliated Hospital of Fujian Medical University. Her research interests include neuropathology and surgical pathology.

Haohua Tu received his BS degree from Tsinghua University, Beijing, China, in 1992 and his PhD from the University of Kentucky, in 2001, both in engineering. He has been a research scientist and a principal investigator at the Biophotonics Imaging Laboratory, University of Illinois at Urbana-Champaign, Urbana. He has 54 peer-reviewed publications, 41 conference proceedings, and multiple patents in the fields of biomedical optical imaging, photonics device development, optical physics, and chemical engineering.

Dezhi Kang is the vice dean of The First Affiliated Hospital of Fujian Medical University, the vice dean of the First Clinical Medical College of Fujian Medical University, and the deputy director of the Fujian Institute of Neurology. He is also a professor and a doctor-student adviser at Fujian Medical University. His expertise is brain tumor surgery treatment of functional encephalopathy.

Jianxin Chen is the director of the Key Laboratory of Optoelectronic Science and Technology for Medicine of Ministry of Education, Fuzhou, China. Her research interests include the development and applications of nonlinear optical microscopy in biological and biomedical research. 